\title{
INCIDENCE AND VIRULENCE TRAITS OF CANDIDA DUBLINIENSIS ISOLATED FROM CLINICALLY SUSPECTED PATIENTS
}

\section{RAJA VINODHINI ${ }^{1}$, KANNAIYAN MOORTHY ${ }^{1,2 *}$, MOHAMMED SALEH AL ABOODY ${ }^{3}$, SURESH MICKYMARAY ${ }^{4}$}

${ }^{1}$ Department of Microbiology, Vivekanandha College of Arts and Sciences for Women (Autonomous), Elayampalayam, Tiruchengode Namakkal, Tamil Nadu, India. ' Department of Microbiology, Wolaita Sodo University, Wolaita Sodo Zone, Ethiopia, Eastern Africa. ${ }^{3}$ Department of Medical Laboratories, Central Bioscience Research Laboratories, College of Science, Al-Zulfi, Majmaah University, Kingdom of Saudi Arabia. ${ }^{4}$ Department of Medical Laboratory, Medical Biotechnology Research Group (MBRG), College of Science, Majmaah University, Kingdom of Saudi Arabia. Email: abbaikannamoorthy@gmail.com

Received: 19 June 2016, Revised and Accepted: 30 June 2016

\section{ABSTRACT}

Objective: Fungal infections are caused by Candida species are increasing mainly in immunocompromised patients. Among Candida species, Candida dubliniensis has recently increasing opportunistic pathogenic yeast. The present study was aimed to assess the incidence and virulence factors of C. dubliniensis isolated from urine samples of various hospitalized clinically suspected patients.

Methods: Totally 1,406 urine samples were processed, among that $9(0.64 \%)$ were identified as $C$. dubliniensis by germ tube production, biochemical test (Candida identification kit), CHROM agar Candida differential medium and growth at $45^{\circ} \mathrm{C}$. Virulence factors of the $C$. dubliniensis, viz., phospholipase, proteinase, esterase, lipase, hemolysin, and biofilm production were detected by standard procedures.

Results: Maximum number of $C$. dubliniensis have ability to produce protienase 6 (66.66\%), followed by esterase 5 (55.55\%), biofilm formation 4 (44.44\%), hemolysin 2 (22.22\%), and phospholipase and lipase 1 (11.1\%), respectively.

Conclusion: The result revealed that these enzymes are potential virulence factors are the most important thing to realize pathogenesis of C. dubliniensis.

Keywords: Candida dubliniensis, Urine, Incidence, Virulence factors.

(C) 2016 The Authors. Published by Innovare Academic Sciences Pvt Ltd. This is an open access article under the CC BY license (http://creativecommons. org/licenses/by/4. 0/) DOI: http://dx.doi.org/10.22159/ajpcr.2016.v9i6.13597

\section{INTRODUCTION}

Fungal pathogens are becoming increasingly important cause of both community-acquired and nosocomial infections; the genus Candida is the most pathogenic fungi [1]. Although Candida is consisting of more than 200 species, only a few of them has been associated with human infection [2]. Of these, C. albicans is the most important infectious agent and represents a serious public health challenge. In recent years, nonalbicans Candida species are also being implicated [3]. In 1995, a new Candida species was identified in human immunodeficiency virus (HIV) infected patient with oropharyngeal candidiasis in Dublin, Ireland [1]. This new species have ability to forms germ tubes and chlamydospores that are almost indistinguishable from those of Candida albicans. Phenotypic characteristics of finding and differentiation of Candida dubliniensis remains problematic in routine identification. On CHROM agar Candida differential medium, $C$. dubliniensis produced the darkgreen colonies within $48 \mathrm{hrs}$ at $37^{\circ} \mathrm{C}$ when freshly isolated from clinical material, but this property is not retained in subculture [4]. Recently, the absence of growth of $C$. dubliniensis at $45^{\circ} \mathrm{C}$ has to be an easy and reliable feature for differentiation of this species from C. albicans [5].

C. dubliniensis has recently been added up to the rising list of potential opportunistic pathogenic yeasts, and it has been recovered from several body sites in many populations; it is most frequently recovered from the oral cavities of HIV-infected patients [6]. Extracellular hydrolytic enzymes seem to play an important role in Candidal overgrowth [7]. The enzymes produced are proteinase, lipase, esterase, and phospholipase; factors which are responsible for the invasiveness and proliferation of fungi caused by the destruction of host tissues, in which the organisms are provided with nutrients by the host tissues [8].
Hemolytic activity is another important virulence factor exhibited by the pathogens which allows growth in the host using several iron-binding proteins as a source of iron. In recent years, there has been a rapid spread of antifungal multidrug resistance, which has become a serious public health problem [9]. Biofilm production was also associated with high-level antifungal drug resistance of the associated organisms [10]. Although extensive studies on epidemiology and phylogeny of $C$. dubliniensis have been performed, little is known about virulence factors such as extracellular enzymes and hemolytic activities [6]. Therefore, the present study was aimed to determine virulence factor (production of proteinase, phospholipase, lipase, esterase, biofilm formation, and hemolytic activity) of $C$. dubliniensis isolated from urine sample.

\section{MATERIALS AND METHODS}

The study included the clinical strains of $C$. dubliniensis recovered from urine samples of various hospitalized clinically suspected patients from Microbiology Department at Doctors Diagnostic Center, Trichy, India. The collected samples were processed in the Department of Microbiology, Vivekanandha College of Arts and Sciences for Women, Namakkal, Tamil Nadu, India. All the isolates were stored with glycerol on deep freezer until further testing.

\section{Urine sample}

A total of 1,406 urine specimens were directly subjected to Gram's staining in addition specimens were routinely cultured on blood agar MacConkey, and cystine lactose electrolyte deficient agar (Himedia, Mumbai, India), for the recovery of microbial growth (bacteria and Candida species). Then, the culture plates were incubated at $37^{\circ} \mathrm{C}$ for 24-48 hrs. After incubation, growth was observed, and suspected colonies 
were inoculated on Sabouraud dextrose agar plates supplemented with chloramphenicol (Himedia, Mumbai, India) and incubated first at $37^{\circ} \mathrm{C}$ for 24-48 hrs to observe the growth of Candida spp.

\section{Mycological investigation}

Then, the isolated colonies were subsequently identified by (i) Germ tube production; (ii) conventional biochemical test using Hi Candida identification kit (Himedia, Mumbai, India); (iii) subculture of two or three representative colonies on CHROM agar Candida differential medium (Himedia, Mumbai, India) and incubation at $37^{\circ} \mathrm{C}$ for $24-48 \mathrm{hrs}$ The Candida isolates were identified by color characteristics on CHROM agar Candida differential medium [11], and (iv) the confirmatory test to differentiate the $C$. dubliniensis from $C$. albicans by growth at $45^{\circ} \mathrm{C}$ [5] The temperature test was performed using Sabouraud's dextrose agar (SDA) with chloramphenicol (Himedia, India). Cultured plates were incubated at $45^{\circ} \mathrm{C}$ and growth was examined daily up to 10 days. For the tests, a standard reference strain of $C$. albicans (ATCC 90028) was used as the control.

\section{Phospholipase activity}

C. dubliniensis isolates were screened for extracellular phospholipase activity by measuring the size of the zone of precipitation after growth on egg yolk agar [12]. The egg yolk medium consisted of 13.0 g SDA,

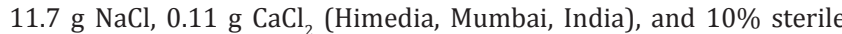
egg yolk (all in $184 \mathrm{ml}$ distilled water). First, these components were mixed and sterilized without the egg yolk; then, the egg yolk was centrifuged at $500 \mathrm{~g}$ for 10 minutes at room temperature, and $20 \mathrm{ml}$ of the supernatant was added to the sterilized medium. Standard inocula of the test and control Candida isolates $\left(5 \mathrm{ml}\right.$, with $10^{8}$ yeast cells $[\mathrm{ml} \text { saline }]^{-1}$ ) were inoculated onto the egg yolk agar medium and left to dry at room temperature. Further, $5 \mathrm{ml}$ of saline, but without yeast cells, was overlaid onto the plate and left to dry at room temperature. Each culture was then incubated at $37^{\circ} \mathrm{C}$ for $48 \mathrm{hrs}$ after which the diameter of the precipitation zone ( $\mathrm{Pz}$ ) around the colony (an indicator of phospholipase activity) was determined. Reference strains of C. albicans (ATCC 90028) served as positive control.

\section{Proteinase activity}

Proteinase production was measured in terms of bovine serum albumin (BSA) degradation according to the technique described by Staib (1965) [13]. The suspension of $1 \times 10^{6}$ cells $\mathrm{ml}^{-1}$ was prepared from Candida isolate. $10 \mu \mathrm{l}$ of suspension was inoculated on $1 \%$ BSA medium plate. The BSA medium consisted of dextrose $2 \%, \mathrm{KH}_{2} \mathrm{PO}_{4} 0.1 \%$, $\mathrm{MgSO}_{4} 0.05 \%$, agar $2 \%$, and $1 \%$ BSA solution (Himedia, Mumbai, India). The plate was incubated for 5 days at $37^{\circ} \mathrm{C}$. After incubation, the plates were fixed with $20 \%$ trichloroacetic acid and stained with $1.25 \%$ amidoblack. Decolorization was performed with acetic acid. Opaqueness of the agar, corresponding to a zone of proteolysis around the colony that could not be stained with amidoblack indicated degradation of the protein. Reference strains of C. albicans (ATCC 90028) served as positive control.

\section{Lipase activity}

Production of lipase was observed by inoculating the isolates of Candida by single diffusion method in SDA agar provided with $1 \%$ tributyrin as substrate (Himedia, Mumbai, India) [14]. Clear zone formation was found around the colony after $24-48 \mathrm{hrs}$ incubation at $37^{\circ} \mathrm{C}$ was considered as lipase activity. Reference strains of $C$. albicans (ATCC 90028) served as positive control.

\section{Esterase activity}

Esterase activity was determined by plate method presented by Slifkin (2000) [15]. The agar medium consisted of $1 \mathrm{~g}$ of peptone, $0.5 \mathrm{~g}$ of $\mathrm{NaCl}$, $0.01 \mathrm{~g}$ of $\mathrm{CaCl}_{2}$, and $1.5 \mathrm{~g}$ of agar (Himedia, India). Medium was sterilized and cooled to about $50^{\circ} \mathrm{C}$, then $0.5 \mathrm{ml}$ of sterile Tween 80 (Himedia, India) was added. An overnight culture of each isolate was inoculated into medium by touching the center of the agar medium with a cotton swab in triplicate. The inoculated culture plates were incubated at $29^{\circ} \mathrm{C}$ and were examined during 10 days. The presence of a halo around a colony was indicative for esterase production by the Candida isolate. Reference strains of $C$. albicans (ATCC 90028) served as positive control.

\section{Biofilm formation}

Biofilm formation was determined for all the isolates, and the standard strains using a method proposed by Branchini et al. (1994) [16] A loopful of colony from the SDA plate was inoculated into $10 \mathrm{ml}$ of Sabouraud's liquid medium supplemented with glucose (final concentration of $8 \%$ ). The tubes were incubated at $37^{\circ} \mathrm{C}$ for $24 \mathrm{hrs}$, after which, the broth was aspirated out and the walls of the tubes were stained with safranin. Biofilm formation was scored as negative $(0)$, weak positive $(1+)$, moderate positive $(2+)$, or strong positive $(3+)$.

\section{Hemolysin activity}

Hemolysin activity of Candida spp. was detected by blood agar plate assay as described by Manns et al. (1994) [17]. The media was prepared by adding $7 \mathrm{ml}$ of aseptically collected fresh sheep blood to $100 \mathrm{ml}$ of SDA supplemented with glucose at a final concentration of $3 \%(w / v) .10 \mu l$ of standard inoculum $\left[10^{8}\right.$ yeast cells $\left.(\mathrm{ml} \text { saline })^{-1}\right]$ prepared from both the test and the control Candida isolates was inoculated onto the medium. The blood agar plate was then incubated at $37^{\circ} \mathrm{C}$ in $5 \% \mathrm{CO}_{2}$ for $48 \mathrm{hrs}$. Hemolysin activity was determined by the translucent zone of hemolysis. Reference strains of C. albicans (ATCC 90028) served as positive control.

\section{Enzymatic score and statistical analysis}

The enzyme activities were expressed as Pz value ( $\mathrm{a}=$ colony diameter/ $\mathrm{b}=$ diameter of the colony plus the precipitation zone) as described by price and co-workers [18]. According to this definition, low $\mathrm{Pz}$ values mean high proteinase or phospholipase and lipase production and inversely, high $\mathrm{Pz}$ values indicate low enzymatic production. The enzymatic activity was scored into four categories: A Pz of 1.0 indicated no enzymatic activity; a Pz between 0.999 and 0.700 indicated low enzymatic activity; Pz between 0.699 and 0.400 corresponded to moderate activity; low $\mathrm{Pz}$ values between 0.399 and 0.100 means high enzymatic activity. Esterase activity (Pz value) was reported as follows: A Pz of 1.0 as negative (-), 0.90-0.99 as weak $(+), 0.80-0.89$ as mild $(++)$, 0.70-0.79 as strong $(+++)$, and $<0.69$ as very strong $(++++)$ [19]. The data were analyzed statistically using mean and standard error of mean.

\section{RESULTS}

A total of 1,406 urine samples were included in the study, among that $9(0.64 \%)$ of the strains were identified as $C$. dubliniensis by following characteristics (i) Germ tube production was detected. (ii) as per the HiCandida identification kit result interpretation, C. dubliniensis strains were negative in urease activity. Maltose, galactose, xylose,

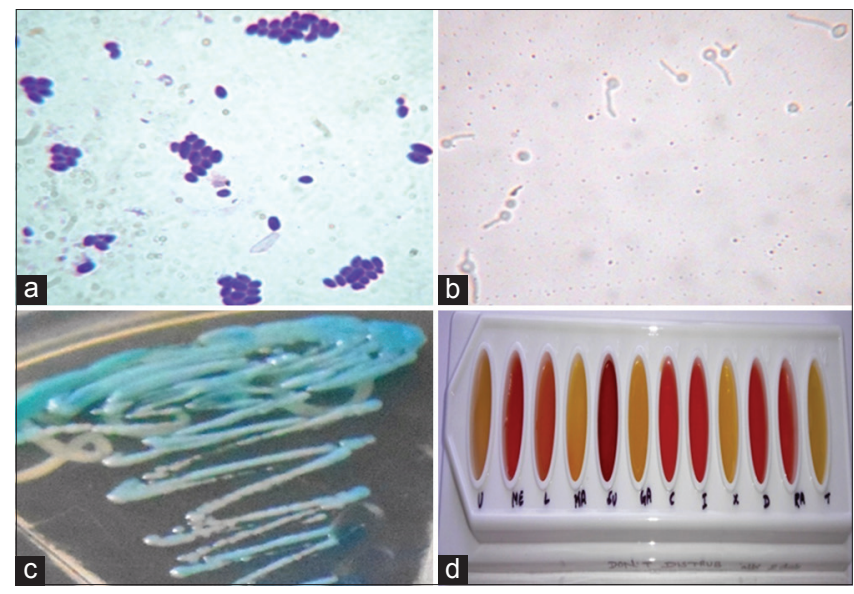

Fig. 1: Mycological investigation of Candida dubliniensis (a) Grampositive budding yeast cells on Gram's staining. (b) Formation of germ tube in human serum at $37^{\circ} \mathrm{C}$. (c) Dark-green colonies on CHROM agar medium. (d) Biochemical test results of Candida dubliniensis on HiCandida identification kit 
and trehalose were utilized. Remaining sugars, viz., melibiose, lactose, sucrose, cellobiose, inositol, dulcitol, and raffinose were not fermented. (iii) dark green colored colonies were observed in CHROM agar Candida differential medium (Fig. 1). (iv) no growth was observed at $45^{\circ} \mathrm{C}$.

Virulence factors of $C$. dubliniensis were demonstrated in Fig. 2. The enzymatic activities of the $C$. dubliniensis (phospholipase, proteinase, esterase, and lipase) were recorded in Table 1. Out of 9 strains phospholipase activity was determined in only one strain $(11.11 \%)$ which showed the moderate activity $(\mathrm{Pz}=0.5$, mean=0.05). Proteinase production was observed in six strains $(66.66 \%), \mathrm{Pz}$ ranged from 0.5 to 0.9 (mean=0.47). Low-level production of esterase was found in five strains (55.55\%), Pz ranged from 0.8 to 0.9 (mean=0.47). Lipase activity was noted as one strain (11.11\%), Pz ranged at 0.9 (mean=0.10) According to the results of enzyme production, maximum number of strains has an ability to release the proteinase and esterase. Table 2 recorded the results of hemolysin and biofilm production. Beta hemolysis was observed in two strains (22.22\%). Biofilm production was noted in four strains (44.44\%).

\section{DISCUSSION}

C. dubliniensis is opportunistic yeast, and it appears to have a worldwide distribution [20,21]. Despite the effort that has been expended to identify $C$. dubliniensis in clinical samples, a definitive assessment of the prevalence of this species is still lacking [2]. C. dubliniensis have

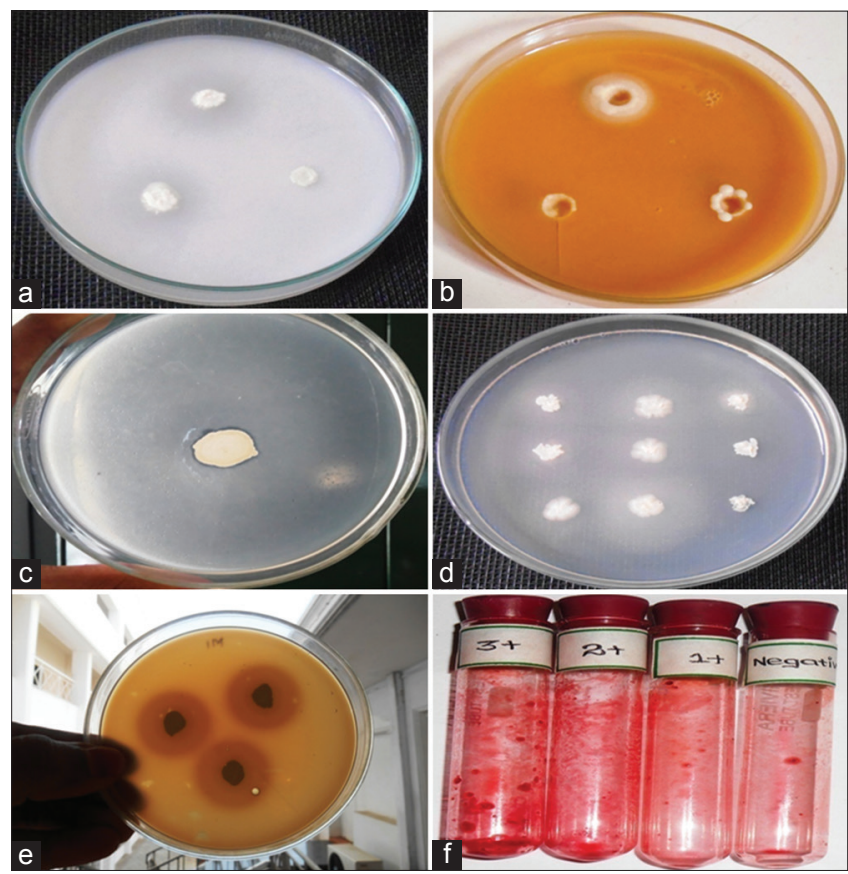

Fig. 2: Virulence factors of Candida dubliniensis (a) Proteinase production on bovine serum albumin medium plate,

(b) Phospholipase production on egg yolk agar, (c) Lipase production on Sabouraud's dextrose agar with $1 \%$ tributyrin oil, (d) Esterase production on Tween 80 opacity medium

(e) Hemolysin production on blood agar plate, and (f) Biofilm formation on Sabouraud's liquid medium with glucose been isolated from a wide range of geographical locations including Europe, North and South America, and Australia [22]. It recovered from several body sites in many populations, but it is most frequently recovered from the oral cavities of HIV-infected patients [6], and 15$30 \%$ has been reported from oral cavities of HIV-infected and AIDS patients [23]. Due to the use of inefficient sampling and identification methods or the sampling on the wrong anatomic sites, the prevalence of C. dubliniensis in normal healthy individuals has been underestimated probably [2]. In a study reported that, on an Irish population of normal healthy individuals, only $3.5 \%$ of individuals were found to carry $C$. dubliniensis in the oral cavity while the prevalence of this species in the vagina was found to be even lower [24] and relatively high prevalence rates of $C$. dubliniensis have been found in oral cavities of patients suffering from diabetes [22]. As well as being associated with oral infections, $C$. dubliniensis has also been identified in a wide range of other anatomic sites [2]. Moreover, isolates of $C$. dubliniensis have been isolated from vaginal, urinary, and fecal specimens [25-27]. Likewise, a study reported that $C$. dubliniensis (31\%) was isolated from urine samples of ICU patients [28]. The percentage of prevalence is very high when compared to our results. In the present study, we identified $9(0.64 \%)$ of $C$. dubliniensis from urine specimens.

Virtually nothing is known about the virulence traits of $C$. dubliniensis. However, it might be expected that this species may share the ability to produce certain putative virulence factors with $C$. albicans [29]. The virulence of $C$. dubliniensis to be low when compared to the virulence of C. albicans. It has been suggested that the reason for the comparatively low virulence is its lower capacity to form hyphae compared to C. albicans [30]. C. dubliniensis is, however, only Candida species in addition to $C$. albicans that forms true hyphae. The expression of a wide range of degradative enzymes, such as secretary aspartyl proteinases (SAPs) and phospholipases has also been recognized as important factors of $C$. albicans to cause infection [2]. C. dubliniensis possesses homologues of seven $C$. albicans secretory aspartyl proteinase genes (SAP) [29]. A prior study reported that proteinase activity of C. dubliniensis resulted in Pz range from 0.61 to 0.84 (mean $=0.75$ ) [6], which is insignificantly low level of enzyme production when compared to the present result. In our study, proteinase production was observed at $66.66 \%$ and $\mathrm{Pz}$ ranged from $0.5-0.9$ (mean=0.47) (Table 1). Bennett et al. (1998) [31] examined that C. dubliniensis possessed sequences homologous to $C$. albicans phospholipase C1 gene (CAPLC1) by southern hybridization and PCR analyses. Sachin et al. (2012) [32] observed $40 \%$ of phospholipase production in C. dubliniensis. However, in our study, only $11.11 \%(\mathrm{Pz}=0.5$, mean=0.05) of phospholipase activity was documented (Table 1 ). The production of phospholipases in $C$. dubliniensis is strain related and the amount produced shows important variations among clinical isolates and depending on the surrounding conditions [33].

Apart from the phospholipases, the term "lipolytic enzymes" comprises lipases and esterases, which hydrolyze glycerol esters of both short- and long-chain fatty acids [34]. The extracellular enzyme described here was considered an esterase, in the strict sense of the word, because of its hydrolytic activity with respect to water-soluble monoesters with a long-chain fatty acid [35]. Esterase activity has been found in a variety of pathogenic Candida species [35-37]. Khosravi et al. (2008) [38] examined esterase activity of $C$. albicans as a potential virulence factor in the animal model. The esterase activities of these yeasts were previously demonstrated in a few studies using the Tween 80 opacity test $[15,35]$.

Table 1: Percentage production of enzymatic activities in C. dubliniensis

\begin{tabular}{llll}
\hline Strain & Enzyme variables & Percentage & Range (Pz) \\
\hline C. dubliniensis $(n=9)$ & Phospholipase & $11.11(1 / 9)$ & 0.5 \\
& Proteinase & $66.66(6 / 9)$ & $0.5-0.9$ \\
& Esterase & $55.55(5 / 9)$ & $0.8-0.9$ \\
\hline
\end{tabular}

SEM: Standard error of mean; Pz: Precipitation zone, C. dubliniensis: Candida dubliniensis 
Table 2: Hemolysin and biofilm production in C. dubliniensis

\begin{tabular}{llll}
\hline Name of the species & Strains name & Hemolysin & Biofilm \\
\hline C. dubliniensis & (n1) & - & $1+$ \\
& (n2) & $\beta$ hemolysis & - \\
& (n3) & - & - \\
(n4) & - & $1+$ \\
(n5) & - & - \\
(n6) & $\beta$ hemolysis & $1+$ \\
$(\mathrm{n} 7)$ & - & $2+$ \\
& (n8) & - & - \\
(n9) & - & - \\
\hline
\end{tabular}

C. dubliniensis: Candida dubliniensis

Similarly, we use the Tween 80 opacity medium for the detection and $55.55 \%$ of esterase activity was determined in C. dubliniensis (Table 1), which is quite high when compare to $16.6 \%$ [39]. Lipase activity of Candida plays a key role in persistence and virulence in humans [40] Lipase is a family of enzymes which hydrolyze the ester bonds in triacylglycerol to yield free fatty acids [41]. In the broad sense of the term, an enzyme which hydrolyzes monoacylglycerol with a long-chain fatty acid (C12 or more) is classified as a monoacylglycerol lipase [36] C. albicans showed higher lipase activity in this study [42]. None of the studies recorded the lipase production in C. dubliniensis. In our study, we observed that $11.11 \%$ of lipase production in $C$. dubliniensis and $\mathrm{Pz}$ ranged at 0.9 (Table 1).

Since there is essentially no free iron in the human host, most pathogens acquire this indirectly from commonly available iron containing compounds such as hemoglobin [43]. The enzymes involved in this activity are classified as hemolysins. Studies on the activity of hemolysin in Candida were limited. Linares et al. (2007) [6] found the hemolytic activity of $C$. dubliniensis. A study observed that $60 \%$ of hemolysin activity in C. dubliniensis [32], which was high when compared to our results $22.22 \%$ (Table 2). Biofilm production is considered to be a potential virulence factor of Candida species. In our study, $44.44 \%$ of $C$. dubliniensis was found as biofilm producers (Table 2). One of the major characteristics of biofilms production is their increased resistance to antifungal drugs. Induction of ergosterol genes has also been described in C. dubliniensis, where incubation with fluconazole and formation of biofilm was coupled with upregulation of the $\mathrm{CdERG3}$ and CdERG25 [44].

\section{CONCLUSION}

In conclusion, our study recommends that an incidence and virulence factor of $C$. dubliniensis from clinical specimens is very important. Although number of epidemiological studies on Candida species, less attention only given to the $C$. dubliniensis. The reason for this may be phenotypic isolation and identification of this species is problematic, which closely related to $C$. albicans. The majority of studies observed the C. dubliniensis in a specific group of populations (HIV and AIDS patients) such studies were failed to record this species from normal healthy individuals. Knowledge about the virulence factors of $C$. dubliniensis is clinically very important. Our study revealed that $C$. dubliniensis had the maximum level of proteinase, esterase activity, biofilm formation and only minimum level of phospholipase, lipase, and hemolytic activity was observed. In addition, prevalence and virulence factors such as esterase, lipase as well as hemolytic activity in $C$. dubliniensis are less studied and importance must be given.

\section{ACKNOWLEDGEMENTS}

The authors are thankful to Prof. M. Karunanithi, Chairman and Secretary, Vivekanandha Educational Institutions, Elayampalayam, and Dr. A. Malarvizhi, Head Department of Microbiology, Vivekanandha College of Arts and Sciences for Women (Autonomous), Elayampalayam, Tiruchengode, Namakkal District, Tamil Nadu for providing all the facilities for our research work.

\section{REFERENCES}

1. Sullivan D, Haynes K, Bille J, Boerlin P, Rodero L, Lloyd S, et al. Widespread geographic distribution of oral Candida dubliniensis strains in human immunodeficiency virus infected individuals. J Clin Microbiol 1998;36:3007-12.

2. Sullivan DJ, Moran GP, Coleman DC. Candida dubliniensis: Ten years on. FEMS Microbiol Lett 2005;253:9-17.

3. Rizvi MW, Malik A, Shahid M, Singhal S. Candida albicans infections in a North Indian tertiary care hospital: Antifungal resistance pattern and role of SDS-PAGE for characterization. Biol Med 2011;3(2):176-81.

4. Schoofs A, Odds FC, Colebunders R, Ieven M, Goossens H. Use of specialised isolation media for recognition and identification of Candida dubliniensis isolates from HIV-infected patients. Eur J Clin Microbiol Infect Dis 1997;16:296-300.

5. Pinjon E, Sullivan D, Salkin I, Shanley D, Coleman D. Simple, inexpensive, reliable method for differentiation of Candida dubliniensis from Candida albicans. J Clin Microbiol 1998;36:2093-5.

6. Linares CE, de Loreto ES, Silveira CP, Pozzatti P, Scheid LA, Santurio JM, et al. Enzymatic and hemolytic activities of Candida dubliniensis strains. Rev Inst Med Trop Sao Paulo 2007;49:203-6.

7. Schaller M, Borelli C, Korting HC, Hube B. Hydrolytic enzymes as virulence factors of Candida albicans. Mycoses 2005;48:365-77.

8. Naglik J, Albrecht A, Bader O, Hube B. Candida albicans proteinases and host/pathogen interactions. Cell Microbiol 2004;6:915-26.

9. Perea S, Patterson TF. Antifungal resistance in pathogenic fungi. Clin Infect Dis 2002;35:1073-80.

10. Ozkan S, Kaynak F, Kalkanci A, Abbasoglu U, Kustimur S. Slime production and proteinase activity of Candida species isolated from blood samples and the comparison of these activities with minimum inhibitory concentration values of antifungal agents. Mem Inst Oswaldo Cruz 2005;100(3):319-23

11. Vijaya D, Harsha TR, Nagaratnamma T. Candida speciation using Chrom Agar. J Clin Diag Res 2011;5(4):755-7.

12. Samaranayake LP, Raeside JM, MacFarlane TW. Factors affecting the phospholipase activity of Candida species in vitro. Sabouraudia 1984;22:201-7.

13. Staib F. Serum-proteins as nitrogen source for yeastlike fungi. Sabouraudia 1965;4:187-93.

14. Aneeja KR. Experiments in Microbiology, Plant Pathology, Tissue Culture and Mushroom Cultivation. II $^{\text {nd }}$ ed. New Delhi: New Age International Publishers; 1996.

15. Slifkin M. Tween 80 opacity test responses of various Candida species. J Clin Microbiol 2000;38(12):4626-8.

16. Branchini ML, Pfaller MA, Rhine-Chalberg J, Frempong $T$, Isenberg HD. Genotypic variation and slime production among blood and catheter isolates of Candida parapsilosis. J Clin Microbiol 1994;32:452-6.

17. Manns JM, Mosser DM, Buckley HR. Production of a hemolytic factor by Candida albicans. Infect Immun 1994;62:5154-6.

18. Price MF, Wilkinson ID, Gentry LO. Plate method for detection of phospholipase activity in Candida albicans. Sabouraudia 1982;20:7-14.

19. Ge YP, Lu GX, Shen YN, Liu WD. In vitro evaluation of phospholipase, proteinase, and esterase activities of Candida parapsilosis and Candida metapsilosis. Mycopathologia 2011;172(6):429-38.

20. Sullivan D, Coleman D. Candida dubliniensis: Characteristics and identification. J Clin Microbiol 1998;36:329-34.

21. Sullivan D, Haynes K, Bille J, Boerlin P, Rodero L, Lloyd S, et al. Widespread geographic distribution of oral Candida dubliniensis strains in human immunodeficiency virus-infected individuals. J Clin Microbiol 1997;35(4):960-4.

22. Willis AM, Coulter WA, Sullivan DJ, Coleman DC, Hayes JR, Bell PM, et al. Isolation of C. Dubliniensis from insulin-using diabetes mellitus patients. J Oral Pathol Med 2000;29:86-90.

23. Pinjon E, Moran GP, Coleman DC, Sullivan DJ. Azole susceptibility and resistance in Candida dubliniensis. Biochem Soc Trans 2005;33(5):1210-4.

24. Pontón J, Rüchel R, Clemons KV, Coleman DC, Grillot R, Guarro J, et al. Emerging pathogens. Med Mycol 2000;38 Suppl 1:225-36.

25. Sullivan DJ, Westerneng TJ, Haynes KA, Bennet DE, Coleman DC. Candida dubliniensis sp. Nov.: Phenotypic and molecular characterization of a novel species associated with oral candidosis in HIV-infected individuals. Microbiology 1995;141:1507-21.

26. Odds FC, Van Nuffel L, Dams G. Prevalence of Candida dubliniensis isolates in a yeast stock collection. J Clin Microbiol 1998;36:2869-73.

27. Quindós G, Carrillo-Muñoz AJ, Arévalo MP, Salgado J, Alonso-Vargas R, Rodrigo JM, et al. In vitro susceptibility of Candida 
dubliniensis to current and new antifungal agents. Chemotherapy 2000;46:395-401.

28. Chaudhary U, Deep A, Chabbra N. Rapid identification and antifungal susceptibility pattern of Candida isolates. Infect Dis Antimicrob Agents 2009;26(2):49-53

29. Gutiérrez J, Morales P, González MA, Quindós G. Candida dubliniensis, a new fungal pathogen. J Basic Microbiol 2002;42(3):207-27.

30. Stokes C, Moran GP, Spiering MJ, Cole GT, Coleman DC, Sullivan DJ. Lower filamentation rates of Candida dubliniensis contribute to its lower virulence in comparison with Candida albicans. Fungal Genet Biol 2007;44(9):920-31.

31. Bennett DE, McCreary CE, Coleman DC. Genetic characterization of a phospholipase $\mathrm{C}$ gene from Candida albicans: Presence of homologous sequences in Candida species other than Candida albicans. Microbiology 1998;144 (1):55-72.

32. Sachin CD, Ruchi K, Santosh S. In vitro evaluation of proteinase, phospholipase and haemolysin activities of Candida species isolated from clinical specimens. Int J Med Biomed Res 2012;1(2):153-7.

33. Hannula J, Saarela M, Dogan B, Paatsama J, Koukila-Kahkola P, Pirinen S, et al. Comparison of virulence factors of oral Candida dubliniensis and Candida albicans isolates in Healthy people and patients with chronic candidosis. Oral Microbiol Immunol 2000;15:238-44.

34. Wilhelm S, Tommassen J, Jaeger KE. A novel lipolytic enzyme located in the outer membrane of Pseudomonas aeruginosa. J Bacteriol 1999;181(22):6977-86.

35. Rudek W. Esterase activity in Candida species. J Clin Microbiol
1978;8:756-9.

36. Casal M, Linares MJ. Contribution to the study of the enzymatic profiles of yeast organisms with medical interest. Mycopathologia 1983;81:155-9.

37. Pospísil L, Kabátová A. Lipolytic activity in some Candida strains (author's transl). Zentralbl Bakteriol Parasitenkd Infektionskr Hyg 1976;131:692-6.

38. Khosravi AR, Riazipour M, Shokri H, Mousavi M. Intracellular esterase activity of Candida albicans and its correlation with pathogenicity in mice. J Med Mycol 2008;18(3):134-40.

39. Kumar CP, Menon T, Sundararajan T, Nalini S, Thirunarayan MA, Rajasekaran S, et al. Esterase activity of Candida species isolated from immunocompromised hosts. Rev Iberoam Micol 2006;23:101-3.

40 Martin S, Borelli C, Korting HC, Hube B. Hydrolytic enzymes as virulence factors of Candida albicans. Mycos 2005;48:365-77.

41. Bier M. Lipases. Methods Enzymol 1955;1:627-42.

42. Ramesh N, Priyadharsini M, Sumathi CS, Balasubramanian V, Hemapriya J, Kannan R. Virulence factors and antifungal sensitivity pattern of Candida Sp. Isolated from HIV and TB patients. Indian J Microbiol 2011;51(3):273-8.

43. Bélanger $M$, Bégin $C$, Jacques $M$. Lipopolysaccharides of Actinobacillus pleuropneumoniae bind pig hemoglobin. Infect Immun 1995;63:656-62.

44. Boreck'a-Melkusova S, Moran GP, Sullivan DJ, Kuchar' 1kov'a S, Chorv'at $\mathrm{D} \mathrm{Jr}$, Bujd'akov'a H. The expression of genes involved in the ergosterol biosynthesis pathway in Candida albicans and Candida dubliniensis biofilms exposed to fluconazole. Mycoses 2009;52(2):118-28. 